\title{
Responsabilidad social universitaria, transferencia y mercadeo tecnológico: vinculación con el entorno social
}

\author{
Martínez de Carrasquero, Cynthia* \\ Rojas de Mavárez, Ligibther** \\ Guillen Romero, Jaqueline*** \\ Antúnez, Nereida****
}

\section{Resumen}

La investigación tuvo como objetivo estudiar acciones de responsabilidad social universitaria (RSU), y de la gestión tecnológica a través de sus componentes: transferencia tecnológica y mercadeo tecnológico, que fortalecen el proceso de vinculación universidad-entorno social. El diseño de investigación es no experimental, con un estudio descriptivo, de campo, la muestra estuvo conformada por 5 dependencias universitarias de la Universidad del Zulia, a cuyos directores se les aplicó un instrumento de preguntas cerradas. En cuanto a los resultados de responsabilidad social, se evidencia que el $75 \%$ de los encuestados manifiestan que las prácticas de RSU son efectivas y eficaces, a pesar de la insuficiencia presupuestaria. La transferencia de tecnología es el servicio mayormente ofertado, participando con un $37 \%$, la cual se mide por actividades de asesorías, cursos de capacitación, talleres, consultorías y asistencia técnica. En cuanto al mercadeo tecnológico se destacan planes de mercadeo, carteras de productos y/o servicios, promoción y publicidad, y generación de ingresos propios. Se constata la participación de la gerencia universitaria a través de actividades clave de RSU, transferencia y mercadeo tecnológico, mediante la proyección de la universidad en el medio productivo y en la búsqueda de generación de ingresos propios, todo ello con la finalidad de promover el incentivo de los actores in-

\section{Recibido: 23-11-11. Aceptado: 12-07-12}

* Profesora Titular de la Universidad del Zulia. Facultad de Ciencias Jurídicas y Políticas. Escuela de Trabajo Social. Economista. Abogada. Magíster en Gerencia de Empresas. Doctora en Ciencias Gerenciales. Post Doctora en Gerencia para la Educación Superior. e-mail: cymart@hotmail.com.

** Profesora Agregada de la Universidad del Zulia. Facultad de Ciencias Jurídicas y Políticas. Adscrita al Centro de Investigaciones en Trabajo Social. Licenciada en Trabajo Social. Magíster en Intervención Social. e-mail: ligibther@hotmail.com.

*** Profesora Asociada de la Universidad del Zulia. Facultad de Ciencias Jurídicas y Políticas. Escuela de Trabajo Social. Licenciada en Trabajo Social. Magister en Intervención Social.

**** Profesora Asociada de la Universidad Cecilio Acosta. Licenciada en Trabajo Social. Magister en Intervención Social. 
volucrados en el proceso de vinculación universidad-entorno social, para la implementación de lineamientos, que sirvan como base para la elaboración de planes de desarrollo.

Palabras clave: Responsabilidad social universitaria, gestión tecnológica, transferencia tecnológica, mercadeo tecnológico, entorno social.

\title{
University Social Responsibility, Transference and Technological Marketing: Connection with the Social Environment
}

\begin{abstract}
The objective of this research was to study the actions of university social responsibility (USR) and technological management through the components: technological transference and technological marketing, which strengthen the university-social environment linking process. The research design is non-experimental, with a descriptive, field study, using a sample consisting of five dependencies at the University of Zulia, to whose directors a closed-question instrument was applied. In terms of social responsibility results, it was shown that $75 \%$ of those interviewed manifested that the USR practices are effective and efficient, despite an insufficient budget. Technology transfer is the main service offered (37\%), which is measured by advising activities, training courses, workshops, consultations and technical assistance. In terms of technological marketing, marketing plans, product and/or service portfolios, promotion and publicity and generating one's own income stand out. The participation of university management is verified through key USR activities, technological transference and marketing, through projecting the university in the productive medium and through the search for personal income generation, all with the purpose of promoting incentive for the actors involved in the university-social environment connection process, to implement guidelines that serve as a basis for creating development plans.
\end{abstract}

Key words: University social responsibility, technological management, technological transfer, technological marketing, social environment.

\section{Introducción}

El papel de las universidades, debe orientarse a la adquisición del conocimiento a partir de un acercamiento al mundo tecnológico y productivo, sin perder de vista una formación humanista que permita internalizar valores. En este sentido, la función social de las universidades influye y transforma su entorno, es decir, es un auténtico factor de transformación social, y que por lo tanto, según sea el fin que se proponga, ayudará a construir una sociedad con unas determi- nadas características, convirtiéndose en un referente y un actor social, que puede promover, y crear capital social. Cada iniciativa social significa creación de una comunidad de aprendizaje, creando una sinergia entre formación, investigación y participación social.

Asimismo, se debe aceptar que hoy el trabajo de la universidad va más allá de sus funciones tradicionales de docencia, investigación y extensión. Ella puede además llegar a influir poderosamente en los modelos y en las orientaciones del desarrollo económico e industrial, 
Responsabilidad social universitaria, transferencia y mercadeo tecnológico...

Martínez, Cynthia; Rojas, Ligibther; Guillen, Jaqueline y Antúnez, Nereida

social y cultural de las regiones donde está inserta (Martínez, 2000). Por lo que se hace imperante, reconocer que la universidad debe proyectar un nuevo rol social y moral antes que asegurar una formación técnica y científica. Ubicándose en el contexto latinoamericano, no todas han transformado totalmente su perfil. Existe una gran encrucijada entre la identidad universitaria y las condiciones éticas, hacia lo que Gibbons (1997) denomina el "modo 2" de generar conocimiento útil socialmente. Sin embargo, en prácticamente todas estas instituciones, lo que se puede observar son nichos, que sin duda alguna constituyen importantes acciones para el cambio en función de un perfil que hoy día se conoce como Responsabilidad Social Universitaria (RSU), teniendo en cuenta que este proceso de RSU debe ser visto como una dinámica ágil de autocrítica y autoaprendizaje, que conduzca a la superación de límites donde las universidades han estado durante mucho tiempo.

En este orden, la RSU compenetra y articula todas las partes orgánicas de la Universidad, incluyendo en una misma estrategia de gestión: la docencia, la investigación y la extensión, ésta última comprende todos los demás servicios universitarios vinculados con el entorno social (pasantías, servicio comunitario, prácticas profesionales, proyectos de extensión, entre otros). Asimismo, Vallaeys (2006) define la RSU como una política de calidad ética del desempeño de la comunidad universitaria (estudiantes, docentes y personal administrativo) a través de los impactos educativos, cognitivos, laborales y ambientales que la universidad genera. El autor citado, manifiesta que la RSU implica una nueva política universitaria: cambia la gestión institucional, el modo de enseñar e investigar, la relación con la sociedad, el conocimiento y el medioambiente, por lo que, obliga a las universidades a pensar en términos de estrategia gerencial, en una gerencia ética e inteligente, lo cual abre paso a un proceso de gestión tecnológica donde la práctica de la educación científica y tecnológica sea vista desde nuevos modelos de enseñanzas, orientados hacia el estímulo de vocaciones en ciencia y tecnología y el desarrollo de las capacidades para la participación pública.

Así, la gestión tecnológica, es concebida por Paredes (1991) "como el conjunto de actividades estratégicas de carácter técnico-gerencial que se implementa en una organización...". Obviamente, trabajar de esta forma en las universidades no es tarea fácil pero tampoco imposible. Se debe empezar por establecer, el reconocimiento de la dinámica institucional, lo cual se torna algo empinado por el escaso diálogo interno en el que conviven las facultades y las dependencias universitarias. Sin embargo, al asumir esta visión de responsabilidad social y de gestión gerencial hacia una institución pertinente, el camino sería mucho más sencillo, obteniendo como resultante, una universidad con pertinencia social donde los resultados de investigación, docencia y extensión puedan ser transferidos a manera de paquetes tecnológicos que generen un alto impacto en el entorno.

Al mismo tiempo, la integración de las funciones universitarias (docencia, investigación y extensión) permite que los procesos de transferencia de resultados de investigación (servicios, laboratorios, consultoría, licenciamiento de patentes y regis- 
tros, capacitación), se conviertan en oferta o portafolio de servicios tecnológicos.

De este modo, las universidades pueden alcanzar un comportamiento socialmente responsable y contribuir a un mejor posicionamiento de su gestión tecnológica, a través del manejo de cantidad y calidad de una investigación que refuerce la relación entre universidad-entorno social, además del desarrollo de programas de transferencia tecnológica.

Al hacer una realidad este continuo "Ciencia-Tecnología-Educación”, se hace necesario una formación socialmente responsable en cuanto a la formación del recurso humano, el cual debe ser altamente capacitado y críticamente consciente, siendo su propósito la satisfacción de necesidades socioeconómicas del entorno inmediato y del país.

Resaltando en esta investigación el binomio de Responsabilidad social universitaria y gestión tecnológica, el papel de ésta última juega un rol determinante, puesto que es considerada como la gerencia sistemática del proceso de generación de nuevos conocimientos, y como la gerencia del desarrollo del conjunto de destrezas e instrumentos necesarios, para sostener un programa pertinente $y$ sistemático de mejoras en el proceso productivo capaz de conducir a la generación y captación de nuevo conocimiento, la cual considera un sistema constituido por cuatro dimensiones: El mercado tecnológico, la transferencia tecnológica, la protección legal del conocimiento y la negociación tecnológica.

En tal sentido, el objetivo central de la investigación se enfoca en estudiar acciones de Responsabilidad social universi- taria (RSU), y de la gestión tecnológica a través de sus componentes: transferencia tecnológica y mercadeo tecnológico, que fortalezcan el proceso de vinculación universidad-entorno social. Se trabajó con una metodología descriptiva, de campo, con una población representada por las dependencias universitarias de la Universidad del Zulia (LUZ).

Desde el punto de vista metodológi$c o$, el desarrollo de la investigación se enmarca bajo un enfoque cuantitativo, el cual refiere aspectos estadísticos relacionados con las variables estudiadas, responsabilidad social y gestión tecnológica, específicamente. Sobre el diseño, o estrategia para el desarrollo de la investigación, se trabajó con un diseño de campo, pues se toman los datos directamente de la realidad para cerciorarse de las condiciones que se han obtenido. El tipo de investigación es descriptiva, y se aplicó una encuesta a ciertas dependencias universitarias.

Al respecto cabe acotar que la población está representada por veintidós (22) dependencias de LUZ, que desarrollan actividades de vinculación con el entorno social, de la cual se seleccionó una muestra de cinco (5) dependencias, entre ellas: División de Extensión de Agronomía, División de Extensión de Ingeniería, Parque Tecnológico Universitario, Instituto de Investigaciones Petroleras de LUZ y Empresa Rental de Agronomía. En atención a los diferentes criterios, destacan sujetos con las siguientes condiciones:

- Demostrada experiencia de integración con diferentes entidades del sector productivo;

- Disposición de un marco legal para la promoción y realización de activida- 
Responsabilidad social universitaria, transferencia y mercadeo tecnológico...

Martínez, Cynthia; Rojas, Ligibther; Guillen, Jaqueline y Antúnez, Nereida

des de mercadeo y transferencia tecnológica.

- Medio de enlace entre la universidad y el sector productivo, para realizar actividades de extensión y proyectar el desempeño académico de las facultades; y se caracterizan por reunir la mayor producción de bienes y servicios universitarios, con potencialidades de integración hacia el sector productivo y, con funciones explícitas de transferencia y mercadeo.

\section{Responsabilidad social universitaria}

Dentro de este marco de idea, se entiende la responsabilidad social como la práctica de una organización respecto a terceros (Ivancevich et al., 1997). En otras palabras, alude al modo en que la empresa u organización se relaciona e impacta en la sociedad a través de sus prácticas, y a la influencia que la sociedad y las expectativas de los actores sociales tienen sobre ésta.

En la última década, el concepto de responsabilidad social se ha constituido como una tendencia en crecimiento, reflejando en definitiva, el resurgimiento de valores en la sociedad y la manifestación de las organizaciones en involucrarse a través de sus iniciativas con la sociedad civil.

En este orden de ideas, el ámbito universitario ha sido siempre cuna de ideas, proyectos y alternativas para temas que se instalan en las sociedades o en ámbitos específicos de ésta, y requieren de cierto marco de pensamiento para utilizarlos mejor. En el caso de la responsabilidad social, la universidad cumple un rol fundamental en la investigación de políticas para llevar adelante, y en el diseño de campos de acción para incursionar, en el análisis de los marcos establecidos para proponer soluciones alternativas. Si bien la tarea llevada adelante por las distintas fundaciones proporcionan a los estudiosos una amplia perspectiva del manejo e implementación de las acciones de RS, la universidad provee al sector datos y estudios que se transforman en complementos ideales del trabajo de estas fundaciones y empresas que contemplan responsabilidad social empresarial.

Visto así, la universidad no solo debe interpretar esa demanda social, esa necesidad de mercado, sino que debe ser un factor importante para promover el cambio social y el desarrollo del entorno. La participación de la comunidad educativa universitaria en el logro de metas, debe darse en el marco de ese ejercicio responsable del juicio de los actos y del respeto a la libertad, la ética y la tolerancia dentro de una perspectiva de compromiso y pertinencia institucional. Cada día la sociedad demanda de la universidad, mayor participación de los hechos sociales.

Desde este punto de vista, el nuevo enfoque de la universidad debe contribuir decididamente a la solución de los críticos problemas que aquejan a la sociedad. Estos problemas, que deben percibirse a través de la identificación de necesidades sociales, económicas y políticas insatisfechas que requieren soluciones a largo plazo, comprometen la participación de amplios sectores públicos y privados, y sobre todo, nuevos esfuerzos y estilos de cooperación ciudadana (Raga, 1998).

De manera que, al concebir la universidad como organización responsable 
de la creación y difusión del conocimiento, como su principal producto, inquieta en el sentido de cómo debe ser administrada y obliga a conocer de ella el criterio de la pertinencia, para comprobar que los objetivos propuestos por la institución corresponden a los requeridos por la sociedad.

Para estar en sintonía con los nuevos tiempos, la universidad requiere definir la visión integral de sus funciones académicas: docencia, investigación y extensión sobre un nuevo paradigma en el cual uno de sus mejores soportes es la comunicación oportuna como eje transformador que le permita, a través de la creación y difusión del conocimiento lograr la correspondencia entre lo que la sociedad demanda por una parte, y la coherencia interna que debe reinar en la universidad, por la otra, en la búsqueda de la pertinencia social que aspira el contexto de las instituciones de educación superior, tratando de hacer compatible el discurso con la acción (Raga, 1998).

Asimismo, la búsqueda del conocimiento, su transmisión y aplicación dan origen a estas funciones básicas de las instituciones universitarias: investigación, docencia y extensión. Si bien, en determinados momentos de su desenvolvimiento histórico una de esas funciones ha prevalecido sobre la otra, el ideal reside en una equilibrada integración de las mismas, de modo que las tres estén siempre presentes en todas las tareas de la actividad universitaria. De este modo, lo deja explícito el plan de desarrollo estratégico y transformación de la Universidad del Zulia (LUZ, 2000-2004), en uno de sus objetivos estratégicos, el cual expresa el fomento de las potencialidades de producción de bienes y servicios competitivos como medio para fortalecer estas funciones básicas, siendo la extensión, una de las funciones motoras como unidad de análisis para el desarrollo de esta investigación. La misma debe dar respuesta a las grandes necesidades de la sociedad y contribuir a la excelencia académica con compromiso social.

En este sentido, es pertinente aclarar los conceptos de responsabilidad social empresarial (RSE) y responsabilidad social universitaria (RSU). La primera hace alusión a un modo de gestión integral de la empresa, donde las estrategias aplicadas en la última década, han logrado superar poco a poco el enfoque filantrópico que limitaba la participación social de la empresa a un conjunto de obras de caridad para poblaciones necesitadas, promovidas gracias al uso de recursos residuales, pero sin relación intrínseca con la actividad productiva cotidiana de la organización.

De lo anterior, se afirma que la Responsabilidad Social es un modo de gestión integral de la empresa, que se puede caracterizar como gestión de impactos (humanos, sociales y ambientales), que la actividad de la organización genera, en un esfuerzo constante por abarcar y satisfacer los intereses de todos los afectados potenciales (Vallaeys, 2006).

Las universidades no podían quedarse alejadas de la reflexión sobre responsabilidad social, no solo porque son organizaciones, sino porque además son responsables de formar a los futuros profesionales que laboraran en las empresas, a los futuros ciudadanos que tendrán que promover democráticamente los derechos humanos, y a los futuros funcionarios que tendrán a su cargo el bien común de nuestro mundo. 
Responsabilidad social universitaria, transferencia y mercadeo tecnológico...

Martínez, Cynthia; Rojas, Ligibther; Guillen, Jaqueline y Antúnez, Nereida

Es asi como el interés corporativo, bien entendido no puede dejar de ser sensible a la sociedad en la cual se desarrolla la organización, y reconocer las intersolidaridades, como lo afirma Morin (2006). Por lo tanto, la responsabilidad social es una exigencia ética y una estrategia racional de desarrollo para la inteligencia organizacional y por ende, de las instituciones de educación superior.

Los dos principales propósitos de la Universidad son ante todo la formación humana y profesional (propósito académico) y la construcción-creación de nuevos conocimientos (propósito de investigación), sabiendo que estos dos fines se relacionan estrechamente.

La responsabilidad social compenetra y articula todas las partes orgánicas de la Universidad, incluyendo en una misma estrategia de gestión la administración en cuanto a la docencia, la investigación y la extensión, la cual comprende todos los demás servicios universitarios vinculados con el entorno social (servicios de consultoría, asesorías, asociaciones, y proyección social, oficina de comunicación institucional, entre otros); lo cual dista mucho del modelo de proyección social voluntaria que solo abarca al cuarto rubro de los impactos.

Con base a lo expuesto, este desplazamiento de la atención hacia el entorno social por parte de la universidad (proyectos sociales hacia fuera), hacia la gestión integral de la organización académica, es la clave para comprender lo que puede y debería significar la responsabilidad social universitaria, con miras a desarrollar la calidad de la gestión social y ambiental de ésta.
Atendiendo a estas consideraciones, se plantea que:

La RSU es una estrategia de gestión universitaria, tratando de mantener un enfoque holístico sobre la propia organización universitaria, y concibiendo iniciativas interdisciplinarias (sinergia entre facultades y dependencias universitarias) e interinstitucionales (asociación de varias funciones de la estructura institucional).

El papel de la universidad no es el de sustituir al estado o a las organizaciones no gubernamentales de desarrollo (ONGS), sino de asumir cada vez mejor su rol de formación superior integral con fines éticos y en pro del desarrollo sostenible de su comunidad, precisando no concebir las relaciones entre la universidad y el entorno social en términos de donación o ayuda unilateral.

Visto de esta manera, y nuevamente citando el Plan de Desarrollo Estratégico (2000-2004), en cuanto a los lineamientos de políticas institucionales académicas, en lo que respecta a la extensión, se le concibe como la relación de la universidad con su entorno, partiendo de un equilibrado intercambio que deje un valor agregado social e institucional. De manera que, la universidad asuma como compromiso la participación de esta en el desarrollo social, cultural y científico de la colectividad.

La proyección social es la función que cumple la universidad con el medio externo. Se apoya en la docencia y la investigación para el diseño y desarrollo de proyectos que articulen las experiencias y saberes de la academia con los saberes y experiencias del entorno. En este proceso la universidad reconoce características, cultura, potencialidades, necesida- 
des y demandas del medio externo, así podrá estar dispuesta a abrir múltiples y flexibles formas de interacción con los sectores sociales, con los gobiernos locales y nacionales, con los organismos no gubernamentales, con las organizaciones populares y con el sector productivo (Ponz, 1996).

Ahora bien, la responsabilidad social en un contexto personal e institucional, según Vallaeys (2006), significa un compromiso para identificar y comprender los efectos de las acciones de los seres humanos en el mundo, considerar los impactos sociales, ambientales y económicos, y los puntos de vista de las partes interesadas; es decir, la responsabilidad social consiste en que los seres humanos se cuiden unos a otros mediante y para la construcción de una sociedad más justa y fraterna. En ese sentido, es muy importante definir la responsabilidad social en estos términos: decir lo que se hace (ser transparente en su rendición de cuentas) y hacer lo que se dice (ser congruente con sus pretensiones).

En Venezuela, según el Artículo 2 de la Ley de Universidades, las universidades son instituciones al servicio de la nación y a ellas corresponde colaborar en la orientación de la vida del país mediante su contribución doctrinaria en el esclarecimiento de los problemas nacionales.

Pero, la universidad no puede convertirse en una corriente partidista, ni en una ONG o en una obra de beneficencia. Su labor específica es el saber y a partir de allí ha de prestar una contribución eficaz en el ordenamiento de la sociedad. Ahora bien, la universidad no escapa de la responsabilidad social, y es que en su sentido amplio la universidad representa la universalidad de saberes, conocimientos y búsqueda de verdad, a través de sus actividades de docencia, investigación, extensión.

Para ejercer la responsabilidad social, la Universidad requiere desarrollar las capacidades precisas para posibilitar la satisfacción de las necesidades sociales de los diversos grupos de interés; y por lo tanto, al desarrollar dichas capacidades la universidad se transforma en una organización inteligente socialmente, que actúa con responsabilidad social, y que al hacerlo alcanza legitimidad social.

La responsabilidad social ha sido definida por muchos autores como la capacidad o estrategia de gerenciar ética e inteligente los impactos que genera la organización en su entorno, tanto interno como externo en la dimensión social, económica, humana y natural. Al respecto, el Proyecto Universidad Construye País (2006) advierte que la universidad debería preocuparse por la forma en gestionar y gerenciar los impactos tanto positivos como negativos que genera a nivel interno (espacio físico como comunidad académica o comunidad de aprendizaje integrada por estudiantes docentes, funcionarios, directivos y egresados) y a nivel externo (entorno económico, social, político, cultural y ambiental) que en muchos casos trasciende las fronteras locales y regionales generando un efecto global.

La Universidad, como organización, genera impactos tanto a las personas que intervienen en ella (administrativos, docentes, estudiantes) como en su entorno social. En este sentido, Vallaeys (2006) sostiene que la responsabilidad social es el afán de responder, de modo ético e inteligente, por esos impactos, 
Responsabilidad social universitaria, transferencia y mercadeo tecnológico...

Martínez, Cynthia; Rojas, Ligibther; Guillen, Jaqueline y Antúnez, Nereida

para que sean, en la medida de lo posible, positivos y no negativos. Una gestión socialmente responsable cuidará los impactos que la universidad tiene con el medio ambiente, el medio social y los recursos humanos, tratando de minimizar los daños posibles y maximizar todos los impactos positivos, en provecho tanto de la sociedad como de la educación que brinda a sus estudiantes, siendo estos su razón de ser.

Estos impactos de la universidad, se soportan en los principios y valores que orientan y constituyen el contenido de la responsabilidad social universitaria. Los principios y valores son guías para el comportamiento humano, son fundamentales y permanentes en una universidad socialmente responsable, puesto que orientan sus funciones o actividades de docencia, extensión e investigación.

Considerando lo anteriormente dicho, los principios y valores con los que la universidad asume su responsabilidad social se estructuran en un sistema, que se puede ordenar en tres planos: personal, social y universitario.

Cada uno de los principios de una u otra forma consolida el concepto de RSU, en la medida que la Universidad los asuma y practique. En una entrevista, el profesor Vallaeys (2005) sintetiza que el principio rector de la ética de la responsabilidad social es el principio de la ecología de la acción de Morín (2006): "Toda acción escapa cada vez más de la voluntad de su autor a medida que entra en el juego de las inter-retro-acciones del medio en el cual interviene". Una universidad que decide ser socialmente responsable, decide al mismo tiempo empezar a luchar contra la "inteligencia ciega" de la compartamentalización de los conocimientos y carreras en la estructura misma del saber académico, dividido hasta el día de hoy en facultades y departamentos.

\subsection{Componentes de la responsabilidad social universitaria}

El desplazamiento de la atención hacia el entorno social por parte de la Universidad (proyectos sociales hacia fuera), y hacia la gestión integral de la organización académica, es la clave para comprender lo que puede y debería significar la responsabilidad social universitaria, con miras a desarrollar la calidad de la gestión social y ambiental de ésta (Martínez, 2005).

Siguiendo el trabajo teórico de Vallaeys (2006) sobre los impactos específicos que genera la universidad y el deber de gestión ética responsable de estos, se han considerado cinco componentes centrales que constituyen las cinco políticas del desarrollo de una estrategia integral y sustentable para la responsabilidad social universitaria, los cuales sólo algunos de ellos han sido desarrollados en la investigación por lo que se presentan algunos resultados, obtenidos del trabajo de campo realizado:

- Gestión ética y calidad de vida institucional, que permita la mejora continua del comportamiento ético y profesional cotidiano de la comunidad universitaria, a partir de la promoción, en las rutinas institucionales, de valores socialmente responsables. En este aparte, la investigación arrojó que el 
$75 \%$ de los directores encuestados manifiestan que las prácticas de responsabilidad social llevadas a cabo por las dependencias analizadas son efectivas y eficaces, a pesar de las limitaciones que estas puedan tener producto de la insuficiencia presupuestaria.

- Gestión medioambiental responsable que permita a la comunidad universitaria mejorar continuamente en su comportamiento ecológico cotidiano, orientado hacia el uso inteligente y respetuoso del medio ambiente. En lo que tiene que ver con la organización interna de la institución, se refleja en el estudio que el $75 \%$ de los encuestados considera que ésta permite la consecución de actividades.

- Participación social responsable que permita a la comunidad universitaria y sus colaboradores externos mejorar continuamente en su comportamiento social solidario para la promoción del desarrollo humano sostenible. En este componente, un $25 \%$ de las dependencias encuestadas asegura que el impacto que genera la Universidad en las comunidades a través del programa de prácticas profesionales es importante para fortalecer esta necesaria vinculación. Igualmente, en lo que respecta a la participación de colaboradores externos para la promoción del desarrollo humano sostenible, se trabajó en la investigación la dimensión de relaciones interinstitucionales en su indicador de convenios, donde el $50 \%$ de los encuestados respondió que el gobierno a través de la estatal petrolera, ha canalizado recursos para generar impactos en las diferentes áreas atendidas, propiciando un desarrollo endógeno en éstas, a través del diseño y ejecución de programas y proyectos. Un $25 \%$ manifiesta que a nivel gubernamental estas acciones de responsabilidad social se realizan en conjunto con la Corporación de desarrollo de la región zuliana (CORPOZULIA), y el resto expresó que existen proyectos muy puntuales que han nacido de las necesidades de los gobiernos municipales (Alcaldías).

- Formación académica socialmente responsable que permita lograr un perfil del egresado como profesional con aptitudes de solidaridad y responsabilidad social y ambiental, en el marco de una verdadera formación integral e íntegra. En los resultados se observa que el $94 \%$ del personal consultado señala que la Universidad incorpora en sus programas de estudio, las asignaturas de ética profesional, responsabilidad social y desarrollo ambiental; y consideran que la universidad promueve la realización de proyectos sociales con la facilitación de cursos sobre servicio comunitario y/o desarrollo comunitario; y vincula sus programas de estudio con la solución de los problemas del país.

- Investigación socialmente útil y gestión social del conocimiento que permita asegurar la generación y transmisión de conocimientos interdisciplinarios congruentes con el desarrollo humano sostenible, tanto en su temática como en su proceso de construcción y difusión. En esta parte, se observa que el $58 \%$ de 
Responsabilidad social universitaria, transferencia y mercadeo tecnológico...

Martínez, Cynthia; Rojas, Ligibther; Guillen, Jaqueline y Antúnez, Nereida

los consultados manifiestan que la Universidad cuenta con líneas de investigación orientadas al desarrollo económico, social, ambiental y cultural del país; vincula a la comunidad universitaria y sociedad en general en los proyectos de investigación; y realiza constantemente jornadas de investigación, encuentros académicos, eventos y congresos nacionales e internacionales donde se difunden y se realizan intercambios de saberes.

En consideración a lo anteriormente señalado, se asume las cinco políticas principales de la RSU, a saber: una universidad éticamente ejemplar en su vida cotidiana; una universidad ecológicamente ejemplar (campus sostenible); generar comunidades de aprendizaje y desarrollo con actores externos; integrar en las currículas el aprendizaje basado en proyectos sociales; y generar conocimientos socialmente útiles según una agenda de desarrollo concertada con otros actores sociales.

\section{Gestión Tecnológica}

Según Paredes (1996), la gestión tecnológica es una de las actividades clave de la empresa, que le permite servir en forma eficiente y eficaz a sus clientes, enfrentar en forma dinámica a sus competidores tanto internos como externos, mantener un ambiente creativo y participativo de trabajo y garantizar una rentabilidad económica atractiva en el largo plazo.

Las interfaces conceptuales y metodológicas entre la gestión tecnológica y la calidad, no se encuentran aún claramente explicitas en la literatura, lo que dificulta la introducción de la gestión tecno- lógica a las micros y pequeñas empresas de manera asociada al control de calidad.

En el ámbito de las economías actuales, Nelson (1998) citado por Camacho (1998), establece que la gestión tecnológica en tanto vía de introducción de nuevos productos y procesos, debe involucrar cambios que suponen una ruptura relativamente profunda con las formas establecidas de hacer las cosas, con lo cual se crean nuevas capacidades. Es por ello que la gestión tecnológica es un concepto con alto contenido económico y social.

Por su parte, Camacho (1998), señala que para que una nación pueda ser capaz de implantar una gestión tecnológica que materialice la innovación debe procurarse la existencia de los siguientes elementos: existencia de una demanda para la innovación nacional, existencia de una cultura innovadora individual e institucional que desarrolle capacidades tecnológicas y capacidad para atenuar los riesgos inherentes a dicha innovación.

El propósito de la gestión tecnológica es darle respuesta a problemas de calidad proporcionando solución, a través del control de calidad tradicional. Sin embargo, incide y presenta una utilidad limitada a oportunidades de adecuación en el producto a las especificaciones deseadas o de ajustar el proceso, equipos, operaciones, materias primas y otros insumos. Dichas limitaciones son demarcadas por la introducción de la gestión tecnológica en las empresas, por lo tanto, el conjunto de ambas presenta una amplia relación sinergística que permite catalizar innovaciones en los sistemas estratégicos, gerenciales, culturales, organizativos y de infraestructura al interior de la empresa y la vincula con necesidades e 
intereses de proveedores y clientes en una alianza estratégica con refuerzos mutuos, que coadyuvan oportunidades ilimitadas para la aplicación de la gestión tecnológica en las micro y pequeñas empresas del sector industrial.

La gestión tecnológica en los países latinoamericanos implica la atención particular al desarrollo de las capacidades locales que faltan y se estiman necesarias para atender, en formas adecuadas, los requerimientos tecnológicos de la producción. El mismo se hace necesario para dar configuración completa al suministro externo de tecnología posibilitando el ejercicio de una capacidad autónoma de decisión tecnológica.

En tal sentido, la Asociación Latino-lberoamericana de Gestión Tecnológica ALTEC (1992), señala que "la magnitud y complejidad de esta doble tarea de desarrollo y uso de la capacidad tecnológica local, aunada a la escasez de los recursos disponibles para ese fin, exigen una gestión tecnológica particularmente eficaz".

Por tal motivo, la gestión tecnológica aboga la innovación en tecnologías duras, como las relativas a la creación y el desarrollo de nuevos productos (bienes y servicios) y procesos físicos o químicos; y la innovación en tecnologías blandas, como las relativas a nuevas maneras de organizar, dirigir y controlar las actividades de la organización, sea ésta privada, pública o de economía mixta.

En este sentido, es un proceso que permite el manejo de la tecnología desde su desarrollo, también como un proceso que integra funciones y como un proceso de desarrollo de implantación de un enfoque estratégico (Medellín, 1994).
La gestión tecnológica implica entonces, el conjunto de acciones y técnicas que enriquecen el modo de pensar y por ende de actuar de una organización, en el desarrollo de las tareas y funciones, lo cual repercute en el manejo eficiente y eficaz con máximos niveles de rentabilidad. En este sentido, se destaca con una estrategia para dinamizar la relación de la Universidad con su entorno, desde dos líneas de acción: transferencia tecnológica y mercadeo tecnológico.

Asumiendo la gestión desde este punto de vista, a través de la transferencia y el mercadeo tecnológico, definitivamente se conduce a la Universidad a un proceso de cambio, donde ésta gestión tecnológica es concebida como un mecanismo a través del cual se transmite conocimiento de punta a la comunidad, a la empresa, al estado respondiendo a problemas actuales y coyunturales de manera pertinente e innovadora.

\subsection{Transferencia tecnológica}

La transferencia de tecnología puede ser definida por la ALTEC (1992) como el traspaso de un paquete tecnológico o parte de él, desde una unidad u organización hacia otra, con el objeto de que esta última produzca y distribuya bienes y servicios.

La transferencia de tecnología en forma material $\mathrm{y}$, progresivamente, en forma no material, desempeñará un papel cada día más determinante, según las tendencias, en las relaciones de comercio exterior de los países. Abarca la transferencia de tecnologías nuevas y conocidas, de tecnologías altamente desarrolladas y de tecnologías estándar. 
Responsabilidad social universitaria, transferencia y mercadeo tecnológico...

Martínez, Cynthia; Rojas, Ligibther; Guillen, Jaqueline y Antúnez, Nereida

Para determinar el nivel de transferencia de tecnologías, debe considerarse sobre todo su carácter complejo. No es justo considerar la transferencia de tecnologías sólo desde el ángulo de las tecnologías nuevas y sofisticadas, y pasar por alto el amplio campo de transferencia de tecnologías conocidas y probadas.

De acuerdo a los planteamientos de la Comisión Económica para América Latina y el Caribe CEPAL y el Instituto Latinoamericano de Planificación Económica y Social (ILPES), simultáneamente surgen, hoy como antes, nuevos campos de aplicación para tecnologías conocidas que también puede clasificarse como tecnologías estándar. Eso no sólo se refiere al sector de los países en vías de desarrollo sino que es un problema de trascendencia internacional. Por eso, limitar la discusión a un nivel "elitista" de las tecnologías nuevas y sofisticadas no responde a las realidades de la economía internacional.

Para la mayoría de las universidades y centros de investigación, la transferencia tecnológica se define, según la Association of University Technology Managers (AUTM, 2010), como el proceso de transferir de una organización a otra los descubrimientos científicos, con el fin de promover el desarrollo y la comercialización. Esta transferencia se lleva a cabo, por lo general, a través de la firma de acuerdos (o contratos) de concesión de licencias entre las Universidades y las empresas privadas o entidades comerciales de capital público.

En función de lo anterior, se presentan los resultados obtenidos en la dimensión transferencia tecnológica. En esta los elementos que se han constituido en ca- nales de transferencia tecnológica con mayor éxito y que han logrado ser transferidos al sector productivo y al entorno social son la asistencia técnica, las asesorías y los cursos de capacitación con una participación del 19\% cada uno de ellos; la consultoría con $15 \%$ y los resultados de proyectos de investigación con el $12 \%$ evidenciándose la existencia de factores y/o características clave que inciden en la transferencia de tecnología hacia el sector productivo, tales como las alianzas con el sector productivo, la existencia de dependencias universitarias para la transferencia tecnológica y el estímulo-beneficios-para el personal universitario.

Este resultado es respaldado por Macaya (1992), cuando afirma que el proceso de vinculación Universidad-Sector Productivo, el desarrollo y la transferencia tecnológica son oportunidades para viabilizar dicha vinculación, planteando que se puede empezar por la prestación de servicios especializados y programas de capacitación y posteriormente con contratos de consultorías y asistencia técnica en áreas donde la universidad tenga un alto nivel de competencia, igualmente apunta que tal vez el área de mayor oportunidad inmediata para muchas universidades latinoamericanas, sea la vinculación vía la formación de recursos humanos y la educación continua.

Entre los tipos de esfuerzos institucionales asociados con la transferencia tecnológica, destacan: las políticas universitarias dirigidas al sector productivo $(22 \%)$, los contactos formales con el sector productivo (17\%); el conocimiento de necesidades del entorno, la visión gerencial de las autoridades universitarias y los 
contactos informales con el sector productivo, los cuales participan cada uno con el $13 \%$. En cuanto a las acciones gerenciales se destacan la comercialización de la tecnología universitaria, transferir y difundir la tecnología universitaria y la formulación de contratos tecnológicos, los cuales se asumen con un $22 \%$ cada uno.

De manera que, la transferencia tecnológica, es de suma importancia para impulsar la vinculación de la universidad con su entorno, mediante la prestación de servicios, implementación de programas de formación continua, y asistencia técnica en áreas de su competencia, sin embargo, para ello es indispensable revisar la normativa universitaria, plantear políticas de trasferencia tecnológica realmente adaptadas a la realidad, y especializar al personal.

\subsection{Mercadeo Tecnológico}

El mercadeo de un negocio es un factor esencial para la supervivencia de las organizaciones. Para llevar a cabo una dirección exitosa, se necesita planear con cuidado, identificar los resultados requeridos y pensar en organizar y controlar las actividades que harán realidad estos resultados. Al final de cada proceso de mercadeo, se impone una revisión del éxito logrado y cómo se pueden obtener mejores resultados en el siguiente período. De esta manera, se entiende mejor el potencial del negocio, se adquiere más confianza y se aumenta el poder para competir y sobrevivir.

Dentro de lo que es el proceso de planeación de mercadeo, se busca responder cuatro preguntas importantes: ¿qué venderá?, ¿a quién se lo venderá?, ¿cuán- to venderá?, ¿a qué precio venderá?, el plan de mercadeo obtiene las respuestas siguiendo una secuencia lógica: misión de la empresa, ¿hacia dónde quiere que vaya el negocio?; análisis externo: ¿qué oportunidades y dificultades hay en su camino?; análisis interno: ¿cuáles de sus puntos fuertes y débiles influyen en la consecución de este objetivo?; objetivos de estrategia: ¿en qué mercados competirá y con qué base?; operaciones de mercadeo: ¿cuál será el papel que desempeñará cada elemento de la mezcla de mercadeo?; programación: ¿cómo se asegurará que ocurran las actividades necesarias en la secuencia correcta y en el momento oportuno?; presupuesto: ¿cuánto costarán las operaciones de mercadeo? por lo tanto, el plan de mercadeo consiste en usar información del presente para darle forma al futuro.

En relación a esta dimensión, se revela en la investigación que todas las dependencias universitarias analizadas, formulan planes de mercadeo y que para tal fin, consideran acciones estratégicas, entre éstas acciones se destacan: los contactos previos con potenciales clientes participando estos con el $42 \%$, y con el $33 \%$ el conocimiento de la cartera de productos/servicios; utilizando asimismo en su proceso de vinculación con el sector productivo funciones de mercadeo tales como: la ética empresarial, la promoción y publicidad y la planificación del precio; las cuales participan con $20 \%$ cada una de ellas; seguida del estudio de la clientela el cual reporta el 16\%. Mientras que entre los objetivos asociados al mercadeo son fundamentales: la proyección de la universidad en el medio productivo y la búsqueda de generación de ingresos 
Responsabilidad social universitaria, transferencia y mercadeo tecnológico...

Martínez, Cynthia; Rojas, Ligibther; Guillen, Jaqueline y Antúnez, Nereida

propios, valiéndose para tal fin de los medios universitarios comunicacionales como lo es el caso de la emisora radial y del periódico de LUZ.

Por otra parte, en cuanto a los planes de mercadeo tecnológico, los resultados indican que las dependencias universitarias analizadas que se vinculan con el entorno social formulan planes de mercadeo en sus actividades de vinculación Universidad-entorno social; es decir que el $100 \%$ de ellas llevan a cabo el procedimiento referido.

A tal efecto, el Centro Interuniversitario de Desarrollo CINDA (1994), expresa que se hace necesario difundir en forma continua y sistemática el trabajo y los logros alcanzados por la universidad para conocimiento de los potenciales usuarios; así mismo, plantea que la universidad de hoy se puede abocar a estudiar en profundidad las posibilidades de generar recursos a través del trabajo de su especialista y del uso de sus múltiples facilidades (investigación, consultoría, educación, licenciamiento, publicaciones, formación de empresas).

Sin embargo y de manera contradictoria los resultados obtenidos, refieren que existe el desconocimiento del potencial de la oferta tecnológica universitaria, al igual que se presenta la falta de integración con la dependencia universitaria.

En lo que respecta a los medios de comunicación, el $50 \%$ cree que el portafolio de las ofertas extensionista de la Universidad del Zulia, es la manera más efectiva de difundir los productos y servicios que la universidad ofrece, el resto de la población aseguran que a través de prensaluz, y el periódico semestral proyección de LUZ, es la forma más efectiva para dar a conocer los impactos que genera la universidad. Tomando en cuenta que los medios de comunicación se constituyen en verdaderos agentes de transformación social, es a través de los periódicos y revistas y, principalmente, a través de la radio y por la televisión, que la población conoce el país donde vive y recibe informaciones esenciales al ejercicio de la ciudadanía.

En síntesis, el mercadeo tecnológico representa hoy en día una herramienta de gran impacto al desarrollo de las instituciones de educación universitaria, es un espacio de encuentro y promoción de grande transformaciones y por ello exige que las universidades formulen planes de mercadeo, considerando acciones estratégicas y empleando funciones de mercadeo principalmente la ética, la promoción, la publicidad, así como la planificación de su presupuesto.

\section{Conclusiones}

Con respecto a la responsabilidad social, se concluye que la participación de la comunidad educativa universitaria en el logro de metas, debe darse en el marco de un ejercicio responsable del juicio de los actos y del respeto a la libertad, la ética y la tolerancia dentro de una perspectiva de compromiso y pertinencia institucional. La universidad entonces, debe contribuir a la solución de los críticos problemas que aquejan a la sociedad y ellos deben percibirse a través de la identificación de necesidades sociales, económicas, políticas, culturales. Esa sería la verdadera proyección social de la universidad, apoyada en las docencia, investigación y extensión de manera interactiva. 
En este sentido, las prácticas de responsabilidad social coordinadas por las dependencias objeto de estudio, son eficientes y eficaces a pesar de la poca asignación presupuestaria. El impacto que genera la universidad en las comunidades a través del programa de prácticas profesionales fortalece esta vinculación. La participación gremial es mínima en las actividades extensionistas de la universidad, haciendo referencia al gremio profesoral activo y jubilado, lo cual representa una amenaza para esta función universitaria. Al mismo tiempo no existe un voluntariado en apoyo a estas actividades.

En cuanto a la transferencia tecnológica, particularmente los canales de transferencia tecnológica que han logrado ser transferidos al entorno social con mayor éxito son la asistencia técnica, las asesorías, los cursos de capacitación y la consultoría. Las alianzas estratégicas con el referido entorno y el estímulo -beneficios económicos- para el personal universitario son factores clave que inciden en la transferencia de tecnología hacia el sector productivo. Las dependencias universitarias no han desarrollado estructuras internas para la transferencia de tecnología, siendo éstas un factor clave para ello. El entrabamiento de la normativa universitaria y la ausencia de políticas de transferencia tecnológica se constituyen en elementos que obstaculizan el proceso de transferencia tecnológica de la universidad hacia el sector productivo. La duplicación de esfuerzos institucionales y la falta de personal especializado no han permitido una mayor viabilidad del proceso transferencia tecnológica. No existen políticas universitarias definidas en cuanto a transferencia tecnológica.
El mercadeo tecnológico, específicamente lo asociado a la estrategia de mercadeo tecnológico se fundamenta en la proyección de la universidad en el medio productivo y en la búsqueda de generación de ingresos propios.

Los contactos previos con potenciales clientes, el conocimiento de la cartera de producto y/o servicios son aplicados como acciones estratégicas de mercadeo. La ética empresarial, la promoción y la publicidad, la planificación del precio, representan las funciones de mercadeo aplicadas por las dependencias universitarias para vincularse con el entorno social. Las acciones gerenciales utilizadas en la negociación tecnológica en el proceso de vinculación son transferencia y difusión de la tecnología universitaria, la comercialización y la formulación de contratos tecnológicos. Se encuentran presentes situaciones consideradas como la obtención de beneficios mutuos, las estrategias de mercadeo, la definición de modalidades/ canales de transferencia tecnológica y la protección previa del conocimiento científico. Existe desconocimiento en la negociación de contratos y en cuanto a la consecución de financiamiento externo. Se carece de estructura organizativa para negociar.

\section{Referencias Bibliográficas}

Association of University Technology Managers (AUTM) (2010). Estados Unidos.

Asociación Latinoamericana de Gestión Tecnológica (ALTEC) (2011). Aspectos conceptuales y metodológicos de la gestión tecnológica. Editorial DOLVIA. Caracas, Venezuela.

Camacho, Jaime (1998). Incubadoras o viveros de empresas de base tecnológi- 
Responsabilidad social universitaria, transferencia y mercadeo tecnológico...

Martínez, Cynthia; Rojas, Ligibther; Guillen, Jaqueline y Antúnez, Nereida

ca. Documento presentado en el XII Congreso Latinoamericano sobre espíritu empresarial. Costa Rica.

Centro Interuniversitario de Desarrollo (CINDA) (1994). Chile. Informe sobre el sistema de Educación Superior. Chile.

Gibbons, M. (1997). Pertinencia de la educación superior en el siglo XXI. Conferencia Mundial de la Educación Superior. UNESCO, París.

Ivancevich, John; Lorenzi, Meter y Skinner, Steven (1997). Gestión, calidad y competitividad. Mc Graw Hill. Madrid, España.

Macaya, Gabriel (1992). Vinculación de la Investigación Universitaria con el sector productivo. PROTEC_CID UNAM. México.

Martínez, Cynthia (2005). Lineamientos estratégicos de gestión tecnológica en el proceso de vinculación universidad-sector productivo. Editorial EDILUZ. Maracaibo, Venezuela.

Martínez, Eduardo (2000). Estrategias, Planificación y Gestión de Ciencia y Tecnología. Editorial Nueva sociedad. CEPAL-ILPES Caracas, Venezuela.

Medellin, Enrique (1994). Evaluación de la gestión tecnológica desde la perspectiva universitaria. Memorias Seminario Internacional de Postgrado en Planificación, Gestión y Estudio de la Ciencia y Tecnología UNAM. México.

Morín, Edgar (2006). La gestión de los recursos tecnológicos Clásicos COTEC No. 3. Madrid. España.
Palacio Federal Legislativo (1970). Ley de Universidades. Gaceta Oficial No. 1429 Extraordinaria de fecha 08 de septiembre de 1970. Venezuela.

Paredes, Leopoldo (1996). Una contribución teórico-metodológica al estudio de la gestión tecnológica. FUNDACITE. Maracaibo, Venezuela.

Ponz, F. (1996). Espíritu universitario: la educación personalizada en la Universidad AA.VV. Rialp. Madrid, España.

Proyecto Universidad Construye País (2006). Responsabilidad Social Universitaria. Una Manera de ser Universidad. Teoría y práctica en la experiencia chilena. Santiago de Chile. Disponible: http://rsuniversitaria.org/web/ images/stories/memoria/UCP\%20 2006.pdf (Consultado 18.04.12).

Raga, J.T. (1998). Claros y oscuros en el proceso de evaluación de la calidad en las universidades. En experiencias y consecuencias de la evaluación universitaria (estrategias de mejora en la gestión) Michavila, f. (editor). Fundación universidad-empresa. Madrid.

Vallaeys, Francois (2006). Programa de apoyo a iniciativas de Responsabilidad Social Universitaria, Ética y Desarrollo. Banco Interamericano de Desarrollo.

Vallaeys, François (2005). ¿Qué es la Responsabilidad Social Universitaria? Revista PALESTRA, Portal de Asuntos Públicos de la Pontificia Universidad Católica del Perú. 